Public Abstract

First Name:Wenbo

Middle Name:

Last Name:Song

Adviser's First Name:James

Adviser's Last Name:Keller

Co-Adviser's First Name:

Co-Adviser's Last Name:

Graduation Term:SP 2011

Department:Computer Science

Degree:PhD

\title{
Title:AUTOMATED VECTOR-VECTOR AND VECTOR-IMAGERY GEOSPATIAL CONFLATION
}

With the rapid advance of geospatial technologies, the availability of geospatial data from multiple sources has increased dramatically. Integration of multi-source geospatial data can provide insights and capabilities not possible with individual datasets. However, multi-source datasets over the same geographical area are often disparate and do not match well with each other. Accurately integrating geospatial data from different sources is a challenging task. In this dissertation research, we proposed a set of innovative geospatial conflation algorithms to attack the multi-source geospatial integration/conflation problem. We developed a novel snake-based approach to conflate two vector road datasets which has several benefits over traditional conflation methods. Since feature matching is one of the most crucial subtasks of conflation, we proposed a new relaxation labeling-based point matching algorithm to provide an elegant and wellmotivated solution to the conflation problem. For the vector-to-imagery conflation, we presented a comprehensive approach by integrating several vector-based and image-based algorithms including spatial contextual signature extraction, road intersections and terminations extraction, relaxation labeling-based point matching, piecewise rubber-sheeting transformation, and snake-based refinement. Finally we extended our road conflation approach to digital parcel map to make it consistent with high-resolution imagery. The experiments on real world geospatial datasets showed excellent results. 